\title{
WNT Signaling Driven by R-spondin 1 and LGR6 in High-grade Serous Ovarian Cancer
}

\author{
SANGHOON LEE ${ }^{1}$, JOHN JUN ${ }^{2}$, WILLIAM J. KIM ${ }^{2}$, PABLO TAMAYO ${ }^{2}$ and STEPHEN B. HOWELL ${ }^{3}$ \\ ${ }^{1}$ Department of Obstetrics and Gynecology, Korea University College of Medicine, Seoul, Republic of Korea; \\ ${ }^{2}$ Division of Medical Genetics and ${ }^{3}$ Division of Hematology, \\ Department of Medicine, University of California, San Diego, CA, U.S.A.
}

\begin{abstract}
Background/Aim: R-spondins control WNT signaling and RSPO1 and LGR6, two of its receptors, are uniquely expressed at high levels in high-grade serous ovarian cancer (HGSOC). The aim of this study was to assess the interrelations between the expression of the RSPOs and LGRs in HGSOC and in the ovarian surface (OSE) and fallopian tube surface epithelium (FTSE) from which HGSOC arises. Materials and Methods: Analysis of TCGA (HGSOC), CCLE (ovary), and other publicly accessed RNA-Seq data using UC San Diego Computational Cancer Analysis Library (CCAL) to perform differential expression analysis, association studies, and gene set inspection using the single-sample GSEA method. Additionally, we employed multiple publicly available databases including StringDB, Human Protein Atlas, and cBioPortal to aid the investigation. Results: Among normal tissues, expression of RSPO1, LGR5 and LGR6 was highest in the fallopian tube. The relative levels of expression of the RSPOs and LGRs in the OSE and FTSE matched those in HGSOC. RSPOI and LGR6 were highly co-expressed in all three tissues. Gene set enrichment analysis (GSEA) showed that expression of RSPO1 was strongly linked to the enrichment of three separate WNT-driven GO pathways. Analysis of genes that impacted overall survival identified two other immediately adjacent genes that control WNT signaling, KREMEN1 and ZNRF3 whose expression and copy number were coordinately linked. Conclusion: RSPO1 and LGR6 are coordinately expressed in HGSOC and the two normal tissues from which this tumor arises, and their expression is linked to WNT signaling pathways known the control cell fate and proliferation.
\end{abstract}

This article is freely accessible online.

Correspondence to: Stephen B. Howell, MD, Division of Hematology, Department of Medicine, University of California, 3855 Health Science Drive, La Jolla, CA 92093, U.S.A. E-mail: showell@health.ucsd.edu

Key Words: R-spondin 1, spondins, ovarian cancer, wnt signaling.
WNT signaling regulates stem cell function, the fate of the daughter cells as they form tissues, their transformation to the malignant state and the growth and metastasis of the resulting tumors. Execution of these myriad functions requires an extraordinary level of regulatory precision. Tight control of activity in the WNT pathway is mediated primarily by ligands that operate over short physical distances to activate receptor systems in stem cells and their progeny. The interaction of the 19 WNT ligands with the 10 members of the family of frizzled (FZD) receptors provides one level of control, and the interaction of the 4 members of the R-spondin family (RSPO1-4) with 3 members of the LGR receptor family (LGR4-6) and 2 ubiquitin ligase co-receptors (RNF43 and ZNRF3) provides another. The interaction of WNT ligands with the FZD receptors is hypothesized to mediate sustained tonic levels of activity, whereas the interaction of the 4 members of the R-spondin family of ligands serves to amplify and modulate the FZD signal over a wider dynamic range (1). These control systems converge to modulate the transcriptional activity of $\beta$-catenin that drives the expression of multiple genes involved in stem cell function, proliferation and differentiation.

There is now strong evidence that high-grade serous ovarian cancer (HGSOC) arises from both the distal fallopian tube surface epithelium (FTSE) and the ovarian surface epithelium (OSE) as the result of genomic events that activate signal transduction systems and metabolic pathways that sustain the transformed state $(2,3)$. The WNT/ $\beta$-catenin pathway is one of the signal transduction systems that is commonly activated in HGSOC, and the increase in its activity has been linked to the proliferation, migration, metastasis and prognosis of these tumors (4-10). As such, this pathway is of substantial interest in the search for new therapeutic options. Activating mutations in $\beta$-catenin itself, or mutations that disable the degradative complex that limits the levels of $\beta$-catenin, result in increased signal output, but these mutations are rare in HGSOC. There is currently very little information on how activity of the WNT/ $\beta$-catenin pathway is up-regulated in HGSOC. The ability of the 
R-spondins to markedly increase WNT signaling in many cell types, and their important role in stem cell biology, suggests a role for them as drivers of enhanced WNT signaling in HGSOC.

There are $4 \mathrm{R}$-spondins in mammals, and they are of particular interest in part because of their ability to regulate stem cell function in many types of epithelia and cancer (11). After intracellular processing and secretion from the cell, they contain 2 furin-like domains at the $\mathrm{N}$-terminal end and a thrombospondin-like domain followed by a basic domain at the C-terminal end. They bind with varying affinities to the LGR4, LGR5 and LGR6 transmembrane receptors, and one consequence of this binding is reduction in the activity of two ubiquitin ligases, RNF43 and ZNRF3, that are positioned adjacent to the LGRs in the plasma membrane (12). In the absence of RSPO binding to the LGRs, RNF43 and ZNRF3 continuously degrade the receptors of the FZD family. By reducing the ability of RNF43 and ZNRF3 to degrade these receptors, the density of FZD on the cell surface increases along with the magnitude of the signal they generate when bound to an appropriate WNT ligand. However, recent studies indicate that RSPOs can activate downstream GTPases and augment signaling even in the absence of LGR4-6 suggesting the involvement of other types of receptors as well $(13,14)$.

The RSPOs are of particular interest because there is now a large body of evidence from lineage tracing studies in mice that two of their receptors, LGR5 and LGR6, are markers of stem cells in the gut, skin and other epithelia $(11,15)$. Much less is known about stem cells in the OSE or FTSE, but lineage tracing in mice suggests that LGR5 marks a stem cell population in the ovary, and LGR6-expressing cells are found in organoids grown from human fallopian tube epithelium $(2,3,16)$. Using data from The Cancer Genome Atlas (TCGA) we previously observed that HGSOC expresses unusually high levels of LGR5 and LGR6 mRNA. HGSOC also has the highest median expression of RSPO1 mRNA but an unusually low level of RSPO2 mRNA (17). We herein report additional analyses of the expression of RSPO1-4 and LGR4-6 in the FTSE and HGSOC that provide evidence for the RSPO1/LGR6 axis as a driver of increased WNT signaling in HGSOC.

\section{Materials and Methods}

Multidimensional cancer datasets. The cBioPortal for Cancer Genomics (http://cbioportal.org) and The Human Protein Atlas dataset (www.proteinatlas.org) were used to explore, visualize, and analyze multidimensional cancer genomics data. These are open-access resources currently providing data from more than 5,000 tumor samples from 20 cancer studies (18-20). We also used datasets from The Cancer Genome Atlas (TCGA, https://www.cancer.gov/tcga) (21) and Gynecologic Tissue and Fluid Repository (22). The pre-processed dataset containing RNA-seq data of normal FTSE and OSE samples was directly downloaded from a Github repository (https://lawrensonlab.github.io/OvarianRNASeq/index.html).

Network associations. Network associations between proteins were explored using STRING v11.0 (https://string-db.org) (23). The "Kaplan-Meier Plotter" (http://kmplot.com/analysis/index.php? $\mathrm{p}=$ service \& cancer=ovar) was used to analyze the overall survival which was based on a total of 374 cases of ovarian cancer for which RNAseq-based gene expression and clinical data is available (24).

Gene expression analysis and GSEA. Enrichment scores for the corresponding samples were calculated using Single-sample Gene Set Enrichment Analysis (ssGSEA) projection (25). Briefly, gene expression values were rank-normalized from their absolute expression, followed by calculation of an enrichment score of individual samples by evaluating the differences in the empirical cumulative distribution functions of the genes in the gene set relative to the remaining genes. A positive enrichment score denotes significant overlap of the gene set with groups of genes at the top of the ranked list, while a negative enrichment score denotes a significant overlap of the signature gene set with groups of genes at the bottom of the ranked list. These resulting ssGSEA scores for individual samples were used for interpreting gene expression profiles using gene sets from the Molecular Signatures Database (MSigDB, www.msigdb.org) $(26,27)$. In addition to the MSigDB gene sets, the YAP gene set used was derived from a previous study (20). To quantify the degree of association, an information-theoretic measure of information coefficient (IC) was calculated and an empirical permutation test for statistical significance calculations carried out against the expression of specific mRNAs (e.g. RSPO1, LGR6 and KREMEN1.

\section{Results}

RSPO and LGR expression in normal ovary and fallopian tube. Examination of RNA-seq data from the Human Protein Atlas dataset (www.proteinatlas.org), which includes 37 normal tissues, suggests that the expression of RSPOI is usually high in the fallopian tube relative to the ovary and other normal tissues (Figure 1). The expression of the other 3 RSPOs is not distinctively higher in these tissues. Interestingly, the expression of 2 of the best understood RSPO1 receptors (LGR5 and LGR6) is also high in the tube relative to other normal tissues (Figure 1). Although limited by the fact that the RNA-seq was performed using mRNA extracted from whole organs rather than just the epithelial components, this observation led to investigation of the differential expression of the RSPOs and LGRs in HGSOC.

Analysis of RNA-seq data generated from just the OSE and FTSE cells from the ovary and fallopian tube confirmed differential expression of RSPOs and LGRs. We examined the data from 114 samples of selectively harvested OSE cells and 74 samples of normal FTSE collected as part of the Gynecologic Tissue and Fluid Repository (22). Among the OSE and FTSE samples, 43 and 50, respectively, contained data on the LGRs and RSPOs, respectively. This analysis revealed distinct differences in the expression of the RSPOs 

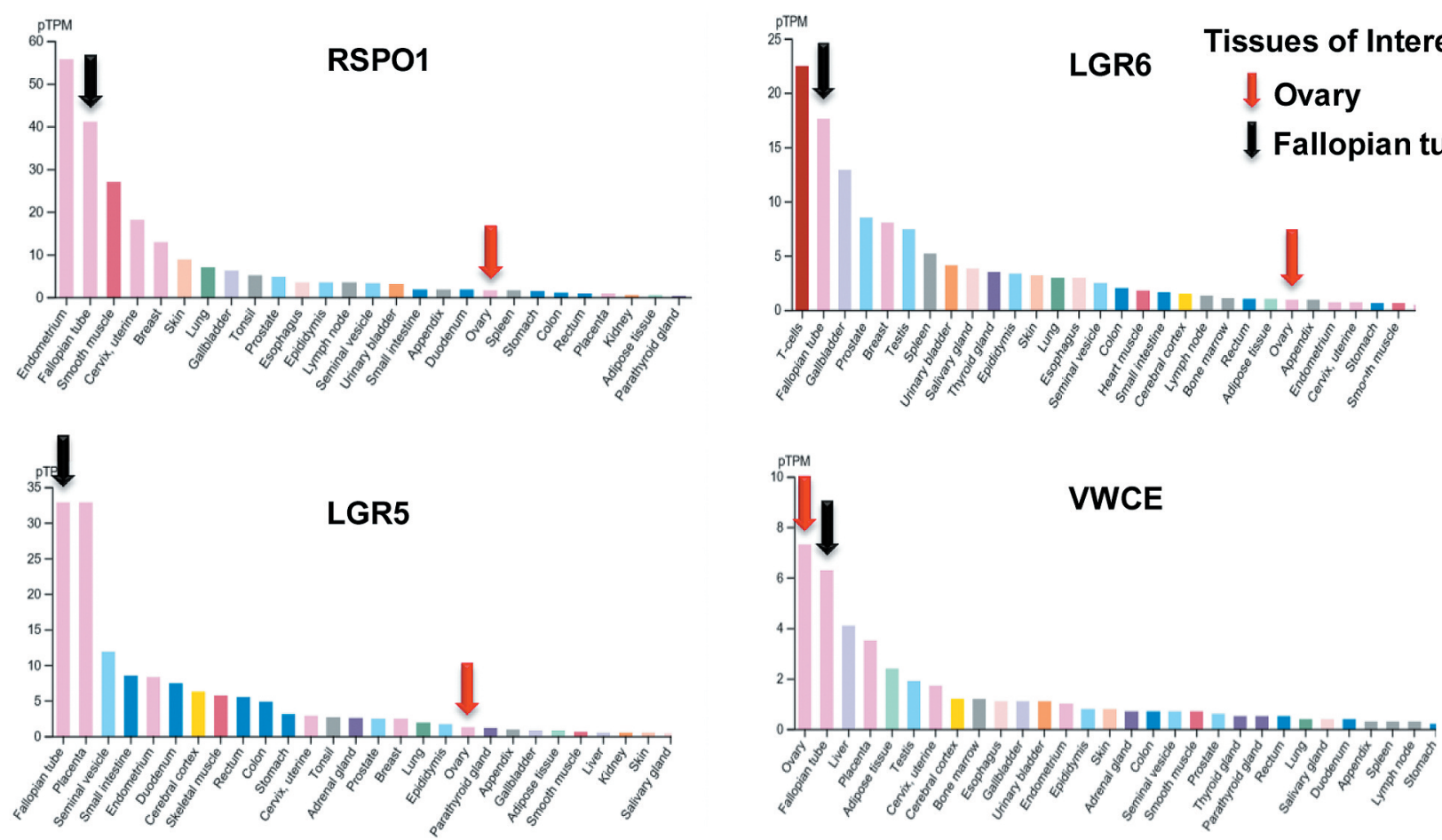

Figure 1. Relative expression of RSPO1, LGR5, LGR6 and VWCE mRNA in normal human tissues. Analysis based on RNA-seq data from the Human Protein Atlas Project.

and LGRs in these epithelia. As shown in Figure 2, in both the OSE and FTSE, RSPO2 stands out as being expressed at a significantly lower level than the other 3 RSPOs. Among the LGRs, LGR4 is expressed at high levels in both tissues, but the OSE and FTSE diverge in the expression of LGR5 and LGR6 both of which are expressed at substantially higher levels in the FTSE than in the OSE. When comparing each of these genes in the FTSE versus OSE it is remarkable that, with the exception of $\mathrm{RSPO4}$, expression is higher in the FTSE than in the OSE.

RSPO and LGR expression in HGSOC. Examination of all of the HGSOC cases in the TCGA database with available RNA-seq data disclosed that the median mRNA level for RSPO1 is among the highest, and that of RSPO2 among the lowest, of all other tumor types in the TCGA database (Figure 3 ). The median levels of expression of $\mathrm{RSPO} 3$ and $\mathrm{RSPO} 4$ did not stand out as being unique in HGSOC. As is the case in normal FTSE, the median level of expression of LGR5 in HGSOC is among the highest observed across all tumor types in TCGA (Figure 4). Also mimicking the FTSE and OSE, expression of $L G R 5$ is higher than LGR6. In contrast to what was observed in FTSE, LGR4 expression in HGSOC was not distinctly elevated relative to other tumor types.
An analysis of the frequency of amplification, mutation, fusion and deep deletion of the 4 RSPOs in the TCGA data showed that the frequency of amplification is higher in HGSOC than in any other tumor type for RSPO1, RSPO2, and $R S P O 4$ (ranging from 4-16\%), but not for RSPO3 (1\%). The frequency of point mutations and deep deletions is low for all 4 RSPOs in HGSOC (3\% for RSPO1 and RSPO2, and $0 \%$ for $\mathrm{RSPO} 3$ and $\mathrm{RSPO} 4$ ). It is also low in almost all other types of tumors. Interestingly, the level of expression of RSPO1 and RSPO2 at the RNA level does not correlate with the degree of gene amplification. No significant difference in the frequency of gene amplification of LGR4-6 was observed in HGSOC compared to other cancers.

We also assessed the impact of these molecular alterations on survival and found no linkage between the expression of RSPO1 or RSPO3 and the overall survival of patients with HGSOC. However, patients with alterations of RSPO2 and/or $R S P O 4$, including amplification, mutation, fusion, and deep deletion, had modest but significant prolongation of overall survival. Median survival in cases with RSPO2 alteration was 25.9 months compared to 44.5 months in cases without alteration $(p=0.0008)$. Median survival was 22.8 months in cases with RSPO4 alteration vs. 44.8 months in cases without alteration $(p=0.045)$. When alterations in either RSPO2 or RSPO4 were considered, median survival was 


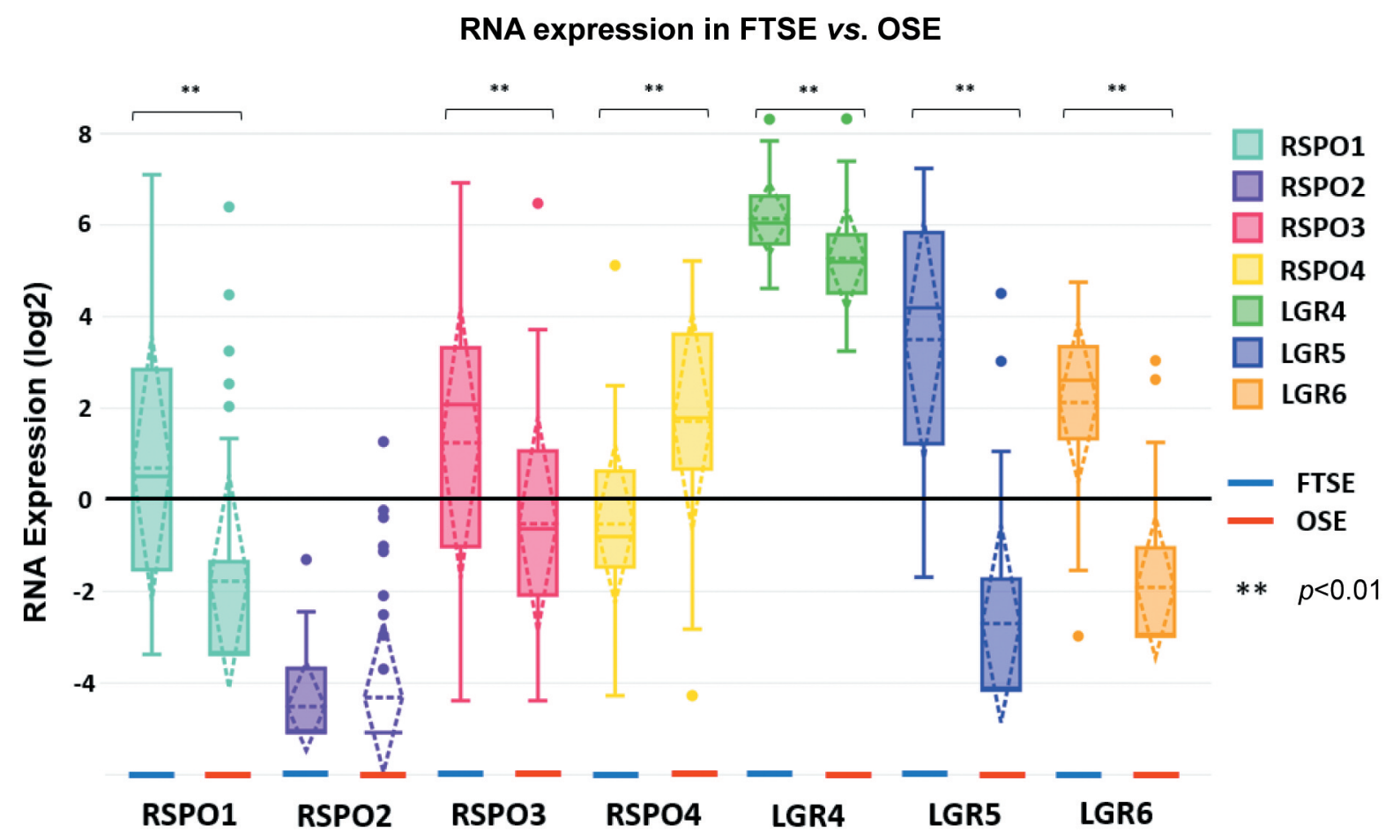

Figure 2. Relative mRNA expression of RSPO1-4 and LGR4-6 in normal OSE and FTSE. Expression in OSE and FTSE determined from RNA-seq data from Lawrenson et al. (22). Box plots indicate median and upper and lower quartiles.

25.9 months compared with 44.9 months in cases without either alteration $(p<0.001)$. However, the number of cases with $R S P O$ alterations in this analysis was small, warranting further analysis using a larger number of cases. The number of cases with either amplification, mutation, fusion, or deep deletion was too small to permit a robust analysis.

Co-expression analysis of RSPO1. Given the prominent expression of RSPO1 in HGSOC, a search was carried out to identify genes whose mRNA levels correlated with that of RSPO1 using the TCGA database. Among 19,007 genes included in the co-expression analysis, AGAP11, LGR6, $A X I N 2$, and $V W C E$ exhibited the highest correlations (Figure $5)$. There were no significant correlations between the expression of RSPO1 and LGR4 or LGR5. While LGR6 and AXIN2 have established roles in the regulation of WNT signaling, AGAP11 and VWCE currently have no known connection to this pathway. However, when AGAP11 was used as the query gene in the co-expression analysis, RSPO1 was found to be the most highly correlated gene suggesting an unknown connection. Also, it is worth noting that mRNA expression of $V W C E$ was very high, and AXIN2 was moderately high, in both the normal ovarian tissue and fallopian tube; no data on the expression of $A G A P 11$ were available in the GTFR database. The significant associations between RSPO1 and LGR6 and AXIN2, and the putative role for AXIN2 vis-a-vis WNT signaling, suggests that RSPO1 participates in driving WNT signaling in HGSOC.

We also analyzed the co-expression of RSPO1, LGR6 and AXIN2 in OSE and FTSE. Table I lists the Spearman correlation coefficients for the association of RSPOI with LGR6 and AXIN2 in the OSE and FTSE; the coefficient for the co-expression of these genes in HGSOC is included for comparison. Figure 6 presents these associations as heat maps. With the exception of the RSPO1-LGR6 association in FTSE, the magnitude of the correlation coefficients in the OSE and FTSE is similar to or greater in the HGSOC consistent with the OSE and FTSE being tissues of origin of HGSOC.

Association between RSPOI and LGR6 expression and GO pathways in HGSOC. To assess the associations of RSPOI and LGR6 at the pathway level, single-sample GSEA (ssGSEA) was used to assess connections of RSPO1 and LGR6 in HGSOC using gene sets from the Molecular Signatures Database (MSigDB, see Methods). As shown in Figure 7, expression of RSPO1 and LGR6 is significantly associated with the enrichment scores of the same 3 separate WNT-related gene sets. Each of these 3 pathways involves components of the canonical WNT signaling pathway $(\beta-$ 


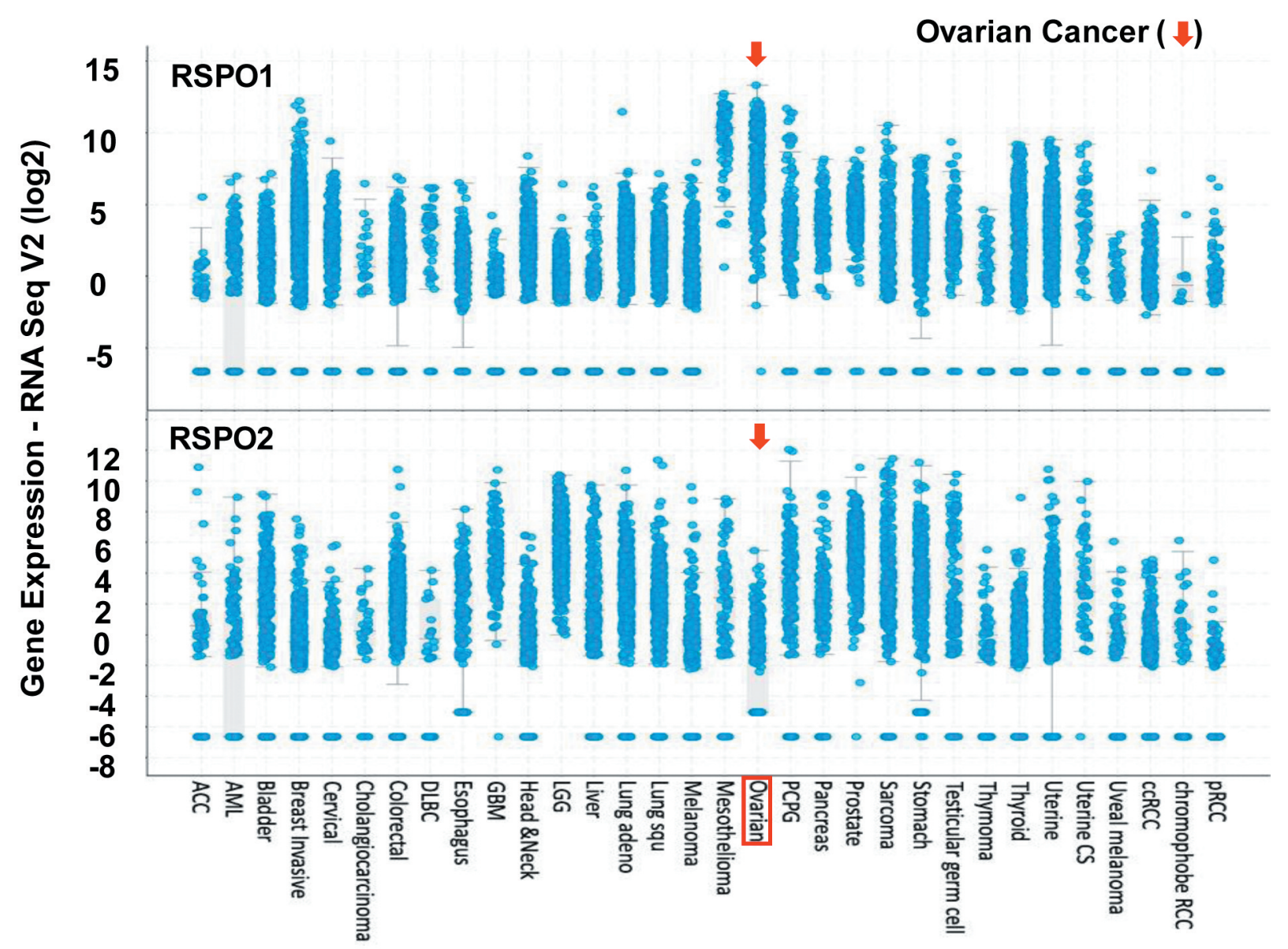

Figure 3. Distribution of mRNA levels of RSPO1 (upper panel) and RSPO2 (lower panel) in HGSOC.

catenin, ER and YAP). This further highlights the close association of RSPO1 with LGR6.

Impact of WNT pathway gene expression on survival in patients with HGSOC. While the expression of RSPO1, LGR5 and LGR6 in HGSOC is associated with WNT pathway signaling, a key question is whether this association influences the clinical behavior of HGSOC. Starting with the list of genes in the WNT STRING cluster (https://string-db.org/) we used the "Kaplan-Meier Plotter" tool (http://kmplot.com/analysis/ index.php? $\mathrm{p}=$ service $\&$ cancer=ovar) to identify those whose expression had the largest effect on progression-free (PFS) and overall survival (OS). This analysis was based on a total of 374 cases of ovarian cancer for which RNA-seq-based gene expression and clinical data was available (28). At the mRNA level none of the RSPOS or LGRS individually had a significant effect on survival. The WNT pathway gene whose expression was most closely associated with a change in PFS and OS was KREMEN1. Although the product of this gene is reported to be an inhibitor of WNT/ $\beta$-catenin signaling (29, 30 ), patients with KREMEN1 expression above the median displayed worse survival; median OS was 40 months for patients with higher expression and 57 months for lower expression $(p<0.001)$ (Figure 8A). ssGSEA identified expression of KREMEN1 to be positively rather than negatively associated to the expression of genes in 2 WNTrelated GO pathways. Two gene sets in MSigDB, representing the activation of the Wnt/ $\beta$-catenin pathway (HALLMARK WNT_BETA_CATENIN_SIGNALING and ST_WNT_BETA _CATENIN_PATHWAY), scored first and 21th respectively out of 6,695 pathways (Figure 8B).

The unexpected observation that increased expression of a putative inhibitor of WNT pathway activation was associated with worse prognosis prompted an analysis of genes co-expressed with KREMEN1 in the HGSOC cases in TCGA. This analysis disclosed a highly positive association with another WNT signaling inhibitor, the ubiquitin ligase ZNRF3 (Spearman 0.5, $p=5.5 \times 10^{-13}, \mathrm{r}^{2}=0.28$ ). In the 


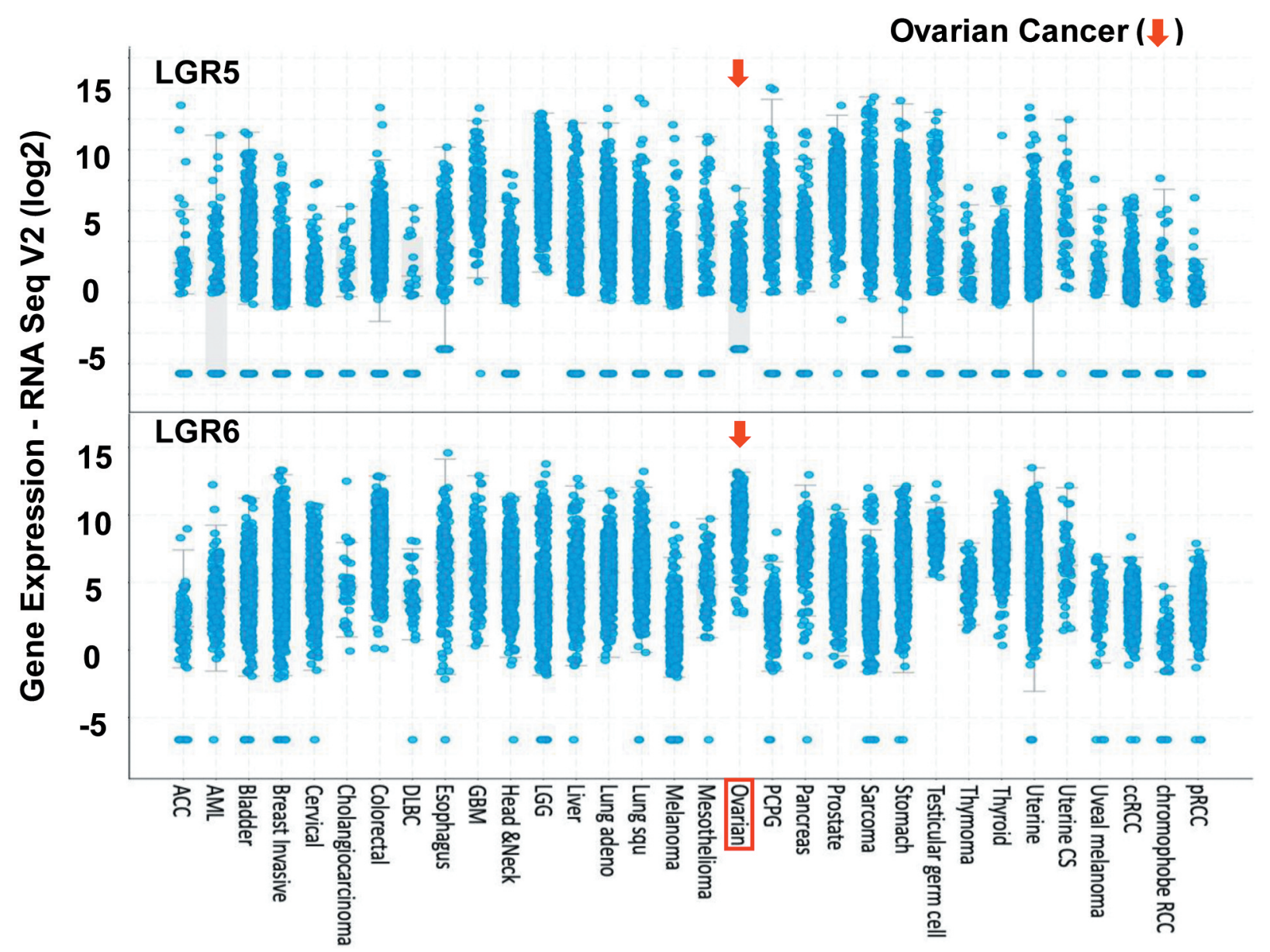

Figure 4. Distribution of mRNA levels of LGR5 (upper panel) and LGR6 (lower panel) in HGSOC.

Table I. Correlations of the mRNA profiles of RSPO1 vs. LGR6 and AXIN2 in OSE, FTSE and HGSOC.

\begin{tabular}{lcccccr}
\hline & \multicolumn{5}{c}{ Spearman correlation coefficient and associated $p$-value } \\
\cline { 2 - 6 } Genes & OSE* $(\mathrm{n}=43)$ & $p$-Value & FTSE* $(\mathrm{n}=50)$ & $p$-Value & HGSOC**(n=299) & $p$-Value \\
\hline RSPO1-LGR6 & 0.54 & 0.0053 & 0.44 & 0.0026 & 0.54 & $5.0 \times 10^{-6}$ \\
RSPO1-AXIN2 & 0.80 & $5.0 \times 10^{-6}$ & 0.51 & $2.9 \times 10^{-4}$ & 0.43 & $4.4 \times 10^{-4}$ \\
\hline
\end{tabular}

*Data source Lawrenson et al. (22); **Data source Weinstein et al. (21). RSPO, R-spondin; LGR, leucine-rich repeat-containing G-protein coupled receptor; OSE, ovarian surface epithelium; FTSE, Fallopian tube surface epithelium; HGSOC, high-grade serous ovarian cancer.

absence of an RSPO, the product of this gene down-regulates WNT signaling by continuously targeting frizzled receptors for proteasomal degradation, an effect that is inhibited by RSPO binding. Interestingly, ZNRF3 lies immediately upstream of KREMEN1 at 22q12.1 suggesting that the positive correlation between the expression of these 2 genes may be the result of co-amplification or co-deletion. To test this hypothesis, the association between copy number of these genes was analyzed (Figure $8 \mathrm{C}$ ). The copy number variation of KREMEN1 and ZNRF3 at 22q12.1 is highly correlated. When KREMEN1 was amplified or deleted, ZNRF3, was also amplified or deleted. The same was true for the other genes in this locus including EMID1 (22q12.2) and TTC28 (22q12.1). Thus, the correlation between the expression of KREMEN1 and ZNRF3 seems likely to be caused by concurrent changes in gene copy number. 


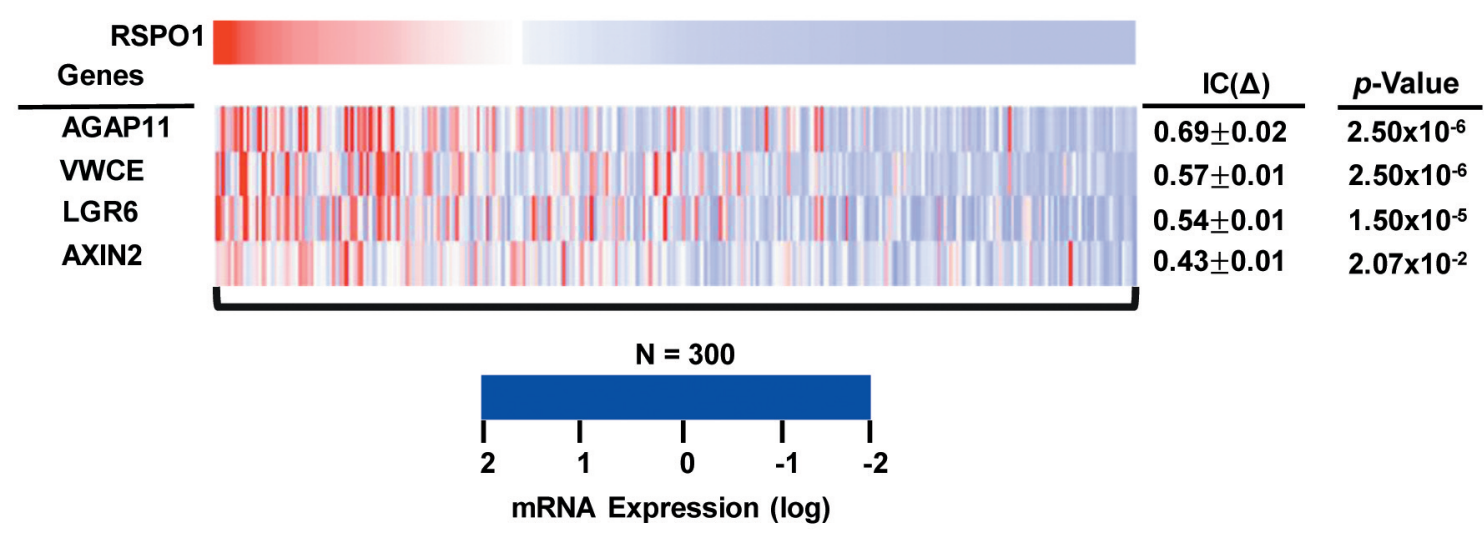

Figure 5. Heatmap showing correlation between the mRNA levels of RSPO1, LGR6, AGAP11, AXIN2, and VWCE in patients with HGSOC. Data are from all 585 cases with RNA-seq data in the TCGA Pan-Caner Atlas study database (21).

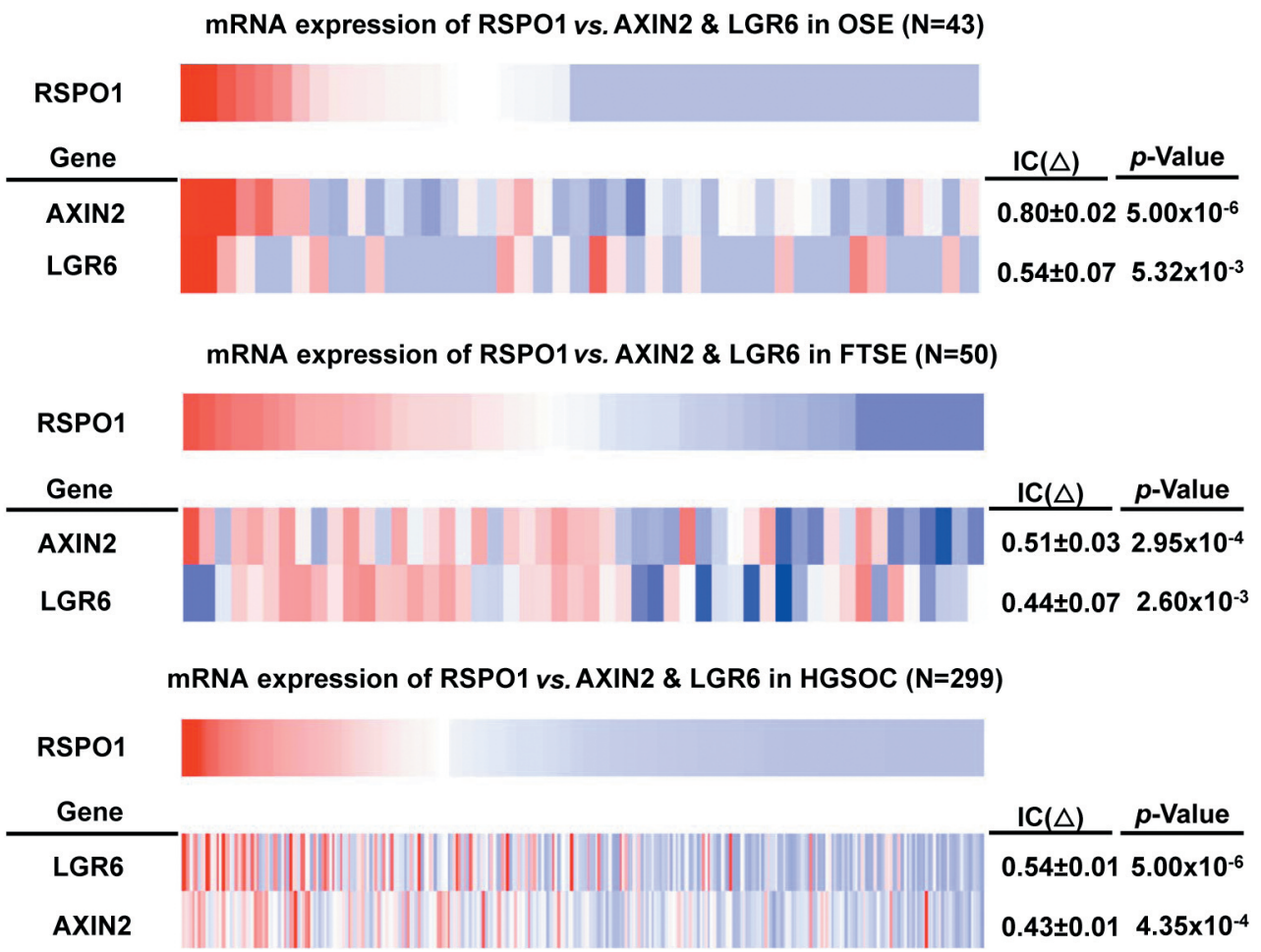

Figure 6. Heatmap showing co-expression in the mRNA levels of RSPO1, LGR6, and AXIN2 in OSE, FTSE, and HGSOC. The Gynecologic Tissue and Fluid Repository (22) dataset was used for analysis of co-expression in OSE and FTSE.

\section{Discussion}

The analyses reported here were prompted by the observation that $R S P O 1$ expression in HGSOC is the second highest among all tumor types, that $R S P O 2$ expression is uniquely low, and that the RSPO1 receptors $L G R 5$ and LGR6 are highly expressed in this malignancy suggesting an important role of WNT signaling as a driver of ovarian cancer. Further analysis disclosed that this pattern of expression is also found in the FTSE and, to a lesser extent, in the OSE, the two epithelia from which HGSOC arises, and that co-expression patterns of RSPOI and LGR6 are similar in these tissues. A comparison of normal tissues in the Human Protein Atlas database disclosed that the fallopian tube expresses RSPO1,LGR5 and LGR6 at higher levels that almost 

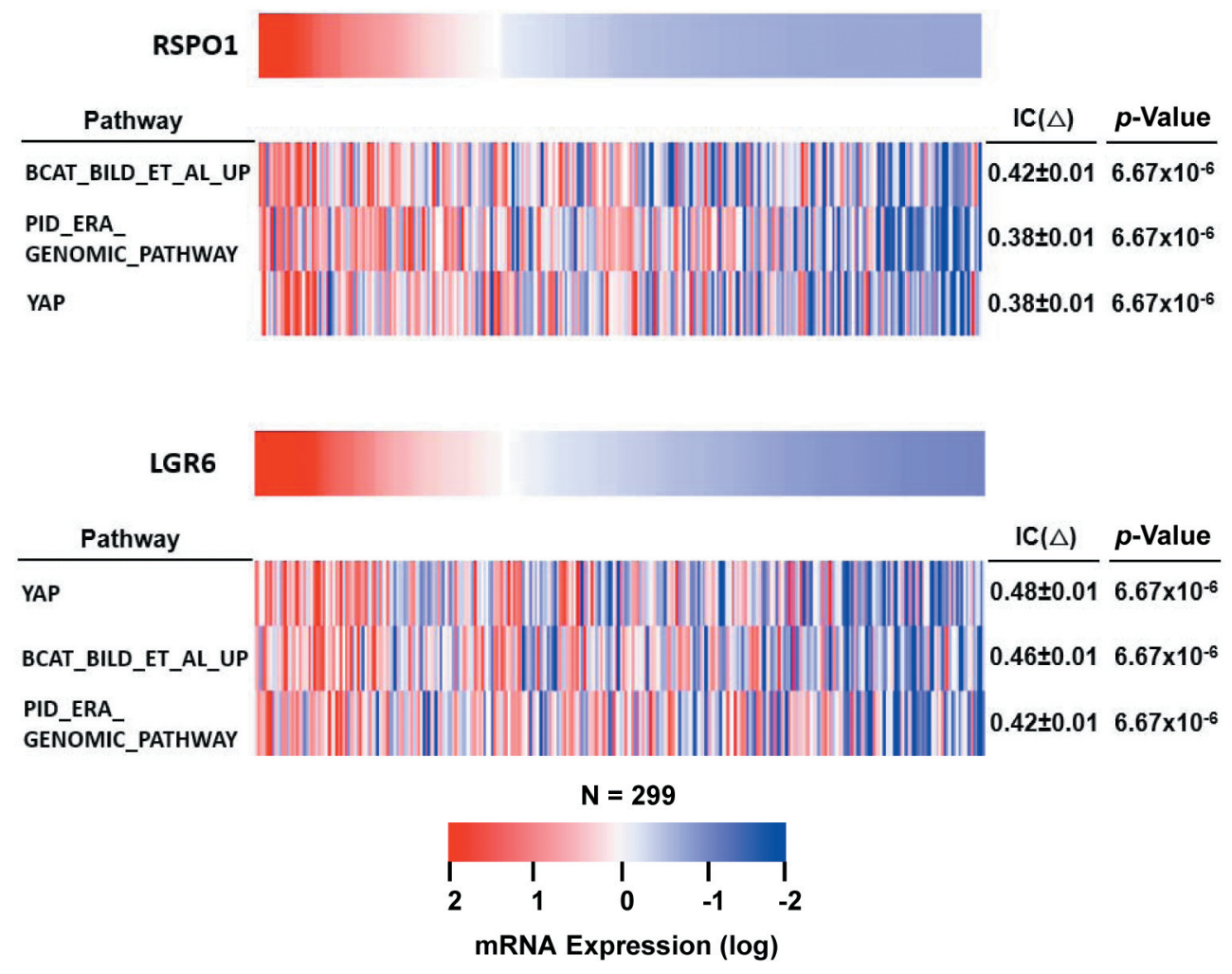

Figure 7. Single-sample GSEA analysis heatmap showing the association between the expression of RSPO1 and LGR6 with WNT-related gene sets in HGSOC.BCAT_BILD_ET_AL_UP: Genes up-regulated in primary epithelial breast cancer cell culture over-expressing activated CTNNB1 gene; PID_ERA_GENOMIC_PATHWAY, genes in the validated nuclear estrogen receptor alpha network; and, YAP, genes associated with YAP activation (HIPPO pathway).

all other tissues. Analysis of RNA-seq data from a large number of non-malignant OSE and FTSE samples confirmed high expression of these 3 genes, and a markedly lower expression of RSPO2, similar to the situation in HGSOC. With the exception of RSPO4, both the RSPOs and LGRs are expressed at lower levels in the OSE than in the FTSE.

Evidence that the high levels of RSPO1 and LGR6 translates into active WNT signaling is provided by the observation that their expression is correlated with that of AXIN2, a gene central to the canonical WNT pathway. GSEA analysis disclosed a strong relationship between expression of either RSPOI or LGR6 and the same 3 WNT-associated pathways ( $\beta$-catenin, ER and YAP). These results are consistent with a prior study that compared RSPO1 mRNA levels in 6 ovarian cancer cell lines relative to immortalized normal human ovarian cells that disclosed 4.5 to 6-fold higher mRNA levels in the malignant cells (4). Strong expression of RSPO1 was detected in 6 human ovarian cancer cell lines by western blot analysis, and all 15 patients whose HGSOC were examined for RSPO1 expression by immunohistochemistry were reported to have strong staining whereas only weak or undetectable staining was found in normal ovaries (4). Thus, within the RSPO family, RSPO1 stands out as a strong but not exclusive candidate driver of WNT signaling in HGSOC. Studies in transgenic mice have clearly established that the increased expression of $R S P O 2$ or RSPO3 that occurs as a result of translocation (EIF3E-RSPO2 and PTPRK-RSPO3) can drive transformation (2). However, such translocations are not found in HGSOC, and whether the increased expression of $\mathrm{RSPOI}$ observed in HGSOC is a cause or consequence of the genomic changes that result in transformation of the OSE or FTSE is unknown.

WNT signaling is required for the growth of organoids derived from both human and mouse OSE and FTSE. Proliferation of cells in these organoids requires RSPO1 (16); whether other RSPOs can substitute for RSPO1 has not been thoroughly tested. However, a recent study of organoids grown from mouse OSE and FTSE disclosed significant differences in the extent to which organoids are dependent on the ligands that support WNT signaling. Organoids grown from OSE have a greater requirement for WNT3A and exhibit differences in marker expression and susceptibility to transformation by known ovarian cancer oncogenes (3). Normal FTSE organoids express $L G R 6$ but not $L G R 5 ; L G R 5$ expression was found to be absent in both the FTSE and organoids and organoids 
A

KREMEN1

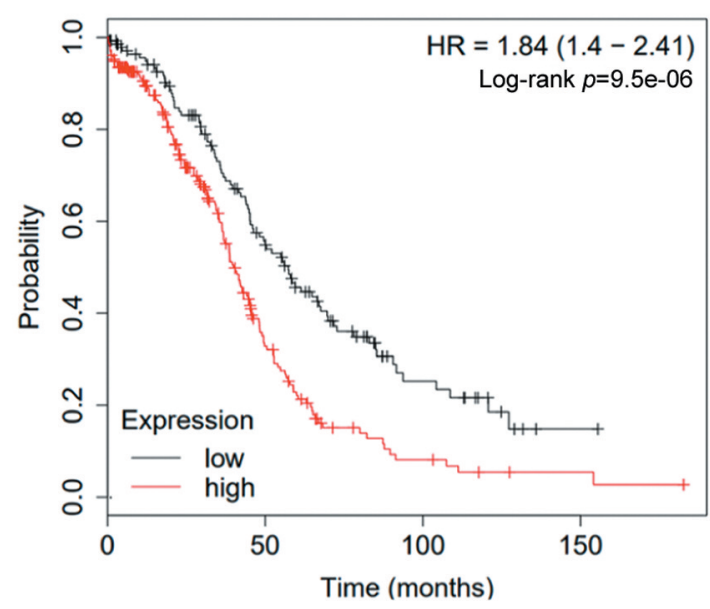

B KREMEN1 Expression vs Wnt-related Pathway Expression in HGSOC (N=300) KREMEN1
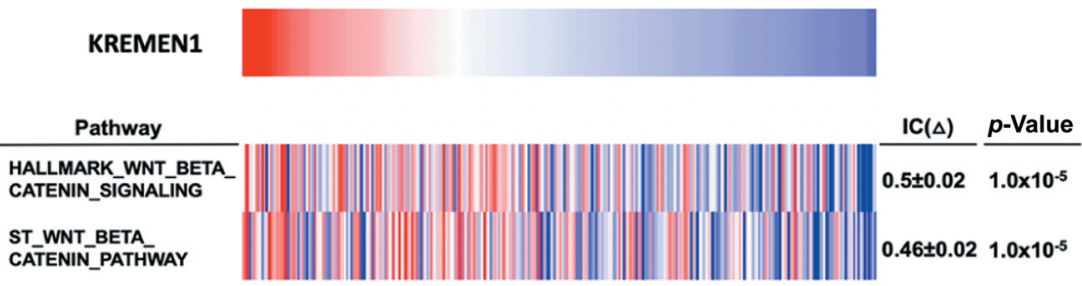

ST_WNT_BETA

$0.46 \pm 0.021 .0 \times 10^{-5}$

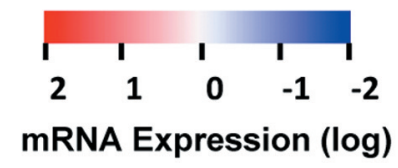

\section{KREMEN1}

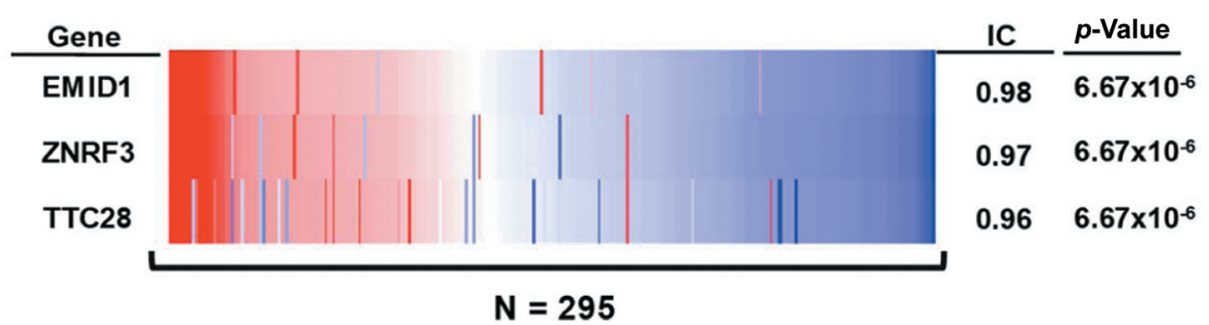

Figure 8. A. Association of the expression of KREMEN1 in patients with HGSOC with overall survival. B. Association of expression of KREMEN1 with WNT signaling pathways in 300 cases of HGSOC (GSEA/MSigDB). C. Linkage between copy number for genes KREMEN1, EMIDI, ZNRF3 and TTC28. Data is from the TCGA Pan-Caner Atlas study database (21).

derived from this epithelium (16). Lineage tracing experiments in mice have recently provided strong evidence that LGR6 marks stem cells in the FTSE whereas LGR5 marks stem cells in the OSE (2). The discrepancy between the results of RNAseq-based analysis of the OSE and FTSE which find high levels of both LGR5 and LGR6 in both the OSE and FTSE likely reflect the cell-type resolution obtainable with organoids.

A search for genes co-expressed with RSPO1 in HGSOC identified LGR6 and AXIN2, both of which are central to WNT signaling. Consistent with the concept that HGSOC relies on signaling pathways dominant in the normal tissue from which it arose, similar co-expression associations were 
found in the OSE and weakly in the FTSE. GSEA analysis indicated that RSPOI and LGR6 are each strongly linked to the same 3 WNT related gene sets which provides evidence of the functionality of the products of these 2 genes in this disease. One of these, the YAP pathway, may be linked to one of the other genes found by the co-expression analysis, $A G A P 11$, a protein-coding gene predicted to have GTPase activity. This finding is of interest for 2 reasons. First, Carmon et al. reported that, in colon cancer cells, LGR5 interacts with the GTPase-activating protein IQGAP1, an effector of Rac1/CDC42 GTPases, to up-regulate actin cytoskeleton dynamics and cell-cell adhesion (31). This establishes a link between RSPO1, LGR5 and GTPase activation in a network that may also involve regulation of the expression of GTPases such as AGAP11. Second, the potential GTPase of activity of AGAP11 provides a possible connection to WNT signaling. Small GTPases act as master organizers of the actin cytoskeleton through activation of YAP/TAZ via switching of their DNA-binding transcription factors (32). YAP/TAZ activation inhibits canonical WNT/ $\beta$ catenin signaling by up-regulating WNT pathway inhibitors DKK1, BMP4, and IGFBP4 (33). The positive co-expression of RSPO1 and AGAP11 suggests that AGAP11 may serve to dampen RSPO1-driven WNT pathway activity. The coexpression analysis disclosed that expression of $V W C E$ was also closely linked to the expression of RSPOI in HGSOC. Under its alias name of URG11 this gene has been reported to drive WNT signaling and promote tumorigenesis in hepatocytes and the gastric epithelium $(34,35)$. We hypothesize that AGAP11 and VCME may both participate in the up-regulation of RSPO1-LGR6-mediated WNT signaling in ovarian cancer.

DDK1 inhibits WNT signaling by interfering with the interaction of WNT ligands to bind to frizzled receptors and the co-receptor LRP. KREMEN1 encodes a high-affinity receptor that binds and disables this function of DDK1 thus augmenting WNT signaling. KREMEN1 likely affects WNT signaling by other means as well as, e.g. in the absence of DKK1, it potentiates $\mathrm{WNT} / \beta$-catenin signaling by maintaining LRP5 or LRP6 at the cell membrane $(29,30)$. The finding that high expression of KREMEN1 is associated with worse OS is consistent the concept that up-regulation WNT signaling supports malignant behavior. However, DDK1 appears to function as a tumor suppressor or promoter in different cellular contexts, and whether and how its interaction with the KREMEN1 protein affects survival in HGSOC through modulation of WNT signaling remains to be further explored.

The fact that the KREMEN1 and ZNRF3 genes are adjacent to each other in the genome, and that changes in their copy numbers are very highly concordant in individual tumors, likely explains their high level of co-expression in HGSOC. How increased levels of ZNRF3 protein might affect the level of WNT signaling in a tumor is not clear since its activity also depends on availability of an RSPO ligand. It is intriguing that a recent study, the GWAS study identified the locus of $R S P O 1$ at $1 \mathrm{p} 34.3$ as a susceptibility locus (36), and even more intriguing that a more recent paper also identified the location of KREMEN1/ZNRF3 as 22q12.1 as a susceptibility locus (37).

Additional support for the hypothesis that RSPO1 and LGR6 are an important divers of WNT signaling in HGSOC comes from in vitro studies of the effect of RSPO1 on normal immortalized human ovarian cells and HGSOC cell lines (4). Addition of recombinant RSPO1 to the medium increased the proliferation and migration of SV-40 Large-T immortalized non-malignant ovarian cells and overexpression increased $\beta$-catenin activity and target gene expression levels. Knockdown of RSPOI expression with siRNA in malignant cells reduced proliferation and migration, reduced $\beta$-catenin activity and target gene expression and induced apoptosis. Immunohistochemical analysis of 294 cases of ovarian cancer disclosed high expression of 1GR6 in 52\% and linked its expression to reduced progression-free and overall survival (10). These data provide evidence the RSPO1/LGR6 axis is functional in quasi-normal ovarian cells, long-established HGSOC cell lines, and clinical samples of HGSOC. However, in vivo evidence that modulating RSPO1 or LGR levels in tumor cells influences growth is not yet available. While RSPO1 and LGR6 stand out as highly expressed at the mRNA level in HGSOC, many of these tumors express the other RSPOs and LGRs at lower levels as well and the level or direction of WNT signaling may well be determined by all of these. As of now there is limited direct in vivo evidence indicating that modulation of RSPO1 protein levels or functional activity can alter WNT signaling or accelerate or impair the growth of this type of tumor.

The major strength of this paper is that it integrates data from many sources, and the databases with which analyses were performed include large numbers of samples that were extensively curated. This permitted us to detect patters of expression, co-expression and linkage to signaling pathways with very high statistical power. The insights reported here now serve to guide efforts to confirm these associations at the experimental level. The fact that this analysis is based on mRNA rather than protein expression is a limitation because of the high variance that exists in the relation between the mRNA and protein level for different genes. It is also a limitation of any study of this type that the databases on which we have relied are heterogenous with respect to patient origin, how mRNA levels were measured, and curation processes.

\section{Conflicts of Interest}

The Authors declare no conflicts of interest. 


\section{Authors' Contributions}

Writing: Original draft preparation, S.L., S.B.H.; review and editing, S.L., S.B.H., J.J., P.T., W.J.K.; conceptualization and visualization, S.L., S.B.H., J.J. and P.T.; supervision, intellectual content, and paper coordination, S.B.H., W.J.K. and P.T.

\section{Acknowledgements}

This project was supported by NIH grants U24CA220341, U01CA217885, U24CA194107, P30 CA023100 and U54CA209891 and the Clayton Foundation for Research. S. Lee is the recipient of grant NRF-2016R1C1B3015250 from the National Research Foundation of Korea.

\section{References}

1 de Lau W, Peng WC, Gros P and Clevers H: The Rspondin/Lgr5/Rnf43 module: regulator of Wnt signal strength. Genes Dev 28(4): 305-316, 2014. PMID: $\quad 24532711$. DOI: $10.1101 / \operatorname{gad} .235473 .113$

2 Zhang S, Dolgalev I, Zhang T, Ran H, Levine DA and Neel BG: Both fallopian tube and ovarian surface epithelium are cells-oforigin for high-grade serous ovarian carcinoma. Nature Commun 10(1): 5367, 2019. PMID: 31772167. DOI: 10.1101/481200

3 Lohmussaar K, Kopper O, Korving J, Begthel H, Vreuls CPH, van Es JH and Clevers $\mathrm{H}$ : Assessing the origin of high-grade serous ovarian cancer using CRISPR-modification of mouse organoids. Nature Commun 11(1): 2660, 2020. PMID: 32461556. DOI: 10.1038/s41467-020-16432-0

4 Liu Q, Zhao Y, Xing H, Li L, Li R, Dai J, Li Q and Fang S: The role of R-spondin 1 through activating Wnt/beta-catenin in the growth, survival and migration of ovarian cancer cells. Gene 689: 124-130, 2019. PMID: 30572097. DOI: 10.1016/j.gene.2018.11.098

5 Arend RC, Londono-Joshi AI, Straughn JM, Jr. and Buchsbaum DJ: The Wnt/beta-catenin pathway in ovarian cancer: a review. Gynecol Oncol 131(3): 772-779, 2013. PMID: 24125749. DOI: 10.1016/j.ygyno.2013.09.034

6 Arend RC, Londono-Joshi AI, Samant RS, Li Y, Conner M, Hidalgo B, Alvarez RD, Landen CN, Straughn JM and Buchsbaum DJ: Inhibition of Wnt/beta-catenin pathway by niclosamide: a therapeutic target for ovarian cancer. Gynecol Oncol. 134(1): 112-120, 2014. PMID: 24736023. DOI: 10.1016/j.ygyno.2014.04.005

7 Bodnar L, Stanczak A, Cierniak S, Smoter M, Cichowicz M, Kozlowski W, Szczylik C, Wieczorek M and LamparskaPrzybysz M: Wnt/beta-catenin pathway as a potential prognostic and predictive marker in patients with advanced ovarian cancer. J Ovarian Res 7: 16, 2014. PMID: 24499657. DOI: 10.1186/1757-2215-7-16

8 Arend RC, Londono-Joshi AI, Gangrade A, Katre AA, Kurpad C, Li Y, Samant RS, Li P, Landen CN, Yang ES, Hidalgo B, Alvarez RD, Straughn JM, Forero A and Buchsbaum DJ: Niclosamide and its analogs are potent inhibitors of Wnt/betacatenin, mTOR and STAT3 signaling in ovarian cancer. Oncotarget 7(52): 86803-86815, 2016. PMID: 27888804. DOI: 10.18632/oncotarget.13466

9 Boone JD, Arend RC, Johnston BE, Cooper SJ, Gilchrist SA, Oelschlager DK, Grizzle WE, McGwin G, Jr, Gangrade A,
Straughn JM Jr. and Buchsbaum DJ: Targeting the Wnt/betacatenin pathway in primary ovarian cancer with the porcupine inhibitor WNT974: Lab Invest 96(2): 249-259, 2016. PMID: 26658453. DOI: $10.1038 /$ labinvest.2015.150

10 Ruan X, Liu A, Zhong M, Wei J, Zhang W, Rong Y, Liu W, Li M, Qing X, Chen G, Li R, Liao Y, Liu Q, Zhang X, Ren D and Wang Y: Silencing LGR6 attenuates stemness and chemoresistance via inhibiting Wnt/beta-catenin signaling in ovarian cancer: Mol Ther Oncolytics 14: 94-106, 2019. PMID: 31193124. DOI: 10.1016/j.omto.2019.04.002

11 Raslan AA and Yoon JK: R-spondins: Multi-mode WNT signaling regulators in adult stem cells: Int J Biochem Cell Biol 106: 2634, 2019. PMID: 30439551. DOI: 10.1016/j.biocel.2018.11.005

12 Hao HX, Xie Y, Zhang Y, Charlat O, Oster E, Avello M, Lei H, Mickanin C, Liu D, Ruffner H, Mao X, Ma Q, Zamponi R, Bouwmeester T, Finan PM, Kirschner MW, Porter JA, Serluca FC and Cong F: ZNRF3 promotes Wnt receptor turnover in an R-spondin-sensitive manner. Nature 485(7397): 195-200, 2012. PMID: 22575959. DOI: 10.1038/nature 11019

13 Lebensohn AM and Rohatgi R: R-spondins can potentiate WNT signaling without LGRs. eLife 7: e33126, 2018. PMID: 29405118. DOI: $10.7554 /$ eLife. 33126

14 Szenker-Ravi E, Altunoglu U, Leushacke M, Bosso-Lefèvre C, Khatoo M, Thi Tran H, Naert T, Noelanders R, Hajamohideen A, Beneteau C, de Sousa SB, Karaman B, Latypova X, Başaran S, Yücel EB, Tan TT, Vlaminck L, Nayak SS, Shukla A and Reversade B: RSPO2 inhibition of RNF43 and ZNRF3 governs limb development independently of LGR4/5/6. Nature 557(7706): 564569, 2018. PMID: 29769720. DOI: 10.1038/s41586-018-0118-y

15 Clevers H, Loh KM and Nusse R: Stem cell signaling. An integral program for tissue renewal and regeneration: Wnt signaling and stem cell control. Science 346(6205): 1248012, 2014. PMID: 25278615. DOI: 10.1126/science.1248012

16 Kessler M, Hoffmann K, Brinkmann V, Thieck O, Jackisch S, Toelle B, Berger H, Mollenkopf HJ, Mangler M, Sehouli J, Fotopoulou C and Meyer TF: The Notch and Wnt pathways regulate stemness and differentiation in human fallopian tube organoids. Nature Comm 6: 8989, 2015. PMID: 26643275. DOI: 10.1038/ncomms9989

17 Schindler AJ, Watanabe A and Howell SB: LGR5 and LGR6 in stem cell biology and ovarian cancer. Oncotarget 9(1): 13461355, 2018. PMID: 29416699. DOI: 10.18632/oncotarget.20178

18 Cerami E, Gao J, Dogrusoz U, Gross BE, Sumer SO, Aksoy BA, Jacobsen A, Byrne CJ, Heuer ML, Larsson E, Antipin Y, Reva B, Goldberg AP, Sander C and Schultz N: The cBio cancer genomics portal: an open platform for exploring multidimensional cancer genomics data. Cancer Discov 2(5): 401-404, 2012. PMID: 22588877. DOI: 10.1158/2159-8290.CD-12-0095

19 Uhlén M, Fagerberg L, Hallström BM, Lindskog C, Oksvold P, Mardinoglu A, Sivertsson Å, Kampf C, Sjöstedt E, Asplund A, Olsson I, Edlund K, Lundberg E, Navani S, Szigyarto CA, Odeberg J, Djureinovic D, Takanen JO, Hober S, Alm T, Edqvist PH, Berling H, Tegel H, Mulder J, Rockberg J, Nilsson P, Schwenk JM, Hamsten M, von Feilitzen K, Forsberg M, Persson L, Johansson F, Zwahlen M, von Heijne G, Nielsen J and Pontén F: Proteomics. Tissue-based map of the human proteome. Science 347(6220): 1260419, 2015. PMID: 25613900. DOI: 10.1126/science.1260419

20 Kim JW, Berrios C, Kim M, Schade AE, Adelmant G, Yeerna H, Damato E, Iniguez AB, Florens L, Washburn MP, Stegmaier K, Gray NS, Tamayo P, Gjoerup O, Marto JA, DeCaprio J and 
Hahn WC: STRIPAK directs PP2A activity toward MAP4K4 to promote oncogenic transformation of human cells. eLife 9: e53003, 2020. PMID: 31913126. DOI: 10.7554/eLife.53003

21. Cancer Genome Atlas Research Network, Weinstein JN, Collisson EA, Mills GB, Shaw KR, Ozenberger BA, Ellrott K, Shmulevich I, Sander C and Stuart JM: The Cancer Genome Atlas Pan-Cancer analysis project. Nat Genet 45(10): 1113-1120, 2013. PMID: 24071849. DOI: 10.1038/ng.2764

22 Lawrenson K, Fonseca MAS, Liu AY, Segato Dezem F, Lee JM, Lin X, Corona RI, Abbasi F, Vavra KC, Dinh HQ, Gill NK, Seo JH, Coetzee S, Lin YG, Pejovic T, Mhawech-Fauceglia P, Rowat AC, Drapkin R, Karlan BY and Noushmehr H: A study of highgrade serous ovarian cancer origins implicates the SOX18 transcription factor in tumor development. Cell Rep 29(11): 37263735, 2019. PMID: 31825847. DOI: 10.1016/j.celrep.2019.10.122

23 Szklarczyk D, Gable AL, Lyon D, Junge A, Wyder S, HuertaCepas J, Simonovic M, Doncheva NT, Morris JH, Bork P, Jensen LJ and Mering CV: STRING v11: protein-protein association networks with increased coverage, supporting functional discovery in genome-wide experimental datasets. Nucleic Acids Res 47(D1): D607-D613, 2019. PMID: 30476243. DOI: 10.1093/nar/gky1131

24 Nagy A, Lanczky A, Menyhart O and Gyorffy B: Validation of miRNA prognostic power in hepatocellular carcinoma using expression data of independent datasets. Sci Rep 8(1): 9227, 2018. PMID: 29907753. DOI: 10.1038/s41598-018-27521-y

25 Barbie DA, Tamayo P, Boehm JS, Kim SY, Moody SE, Dunn IF, Schinzel AC, Sandy P, Meylan E, Scholl C, Frohling S, Chan EM, Sos ML, Michel K, Mermel C, Silver SJ, Weir BA, Reiling JH, Sheng Q and Hahn WC: Systematic RNA interference reveals that oncogenic KRAS-driven cancers require TBK1. Nature 462(7269): 108-112, 2009. PMID: 19847166. DOI: 10.1038/nature08460

26 Liberzon A, Subramanian A, Pinchback R, Thorvaldsdottir H, Tamayo P and Mesirov JP: Molecular signatures database (MSigDB) 3.0. Bioinformatics 27(12): 1739-1740, 2011. PMID: 21546393. DOI: 10.1093/bioinformatics/btr260

27 Liberzon A, Birger C, Thorvaldsdottir H, Ghandi M, Mesirov JP and Tamayo P: The Molecular Signatures Database (MSigDB) hallmark gene set collection: Cell Syst 1(6): 417-425, 2015. PMID: 26771021. DOI: 10.1016/j.cels.2015.12.004

28 Gyorffy B, Lanczky A and Szallasi Z: Implementing an online tool for genome-wide validation of survival-associated biomarkers in ovarian-cancer using microarray data from 1287 patients. Endocr Rel Cancer 19(2): 197-208, 2012. PMID: 22277193. DOI: 10.1530/ERC-11-0329

29 Mao B, Wu W, Davidson G, Marhold J, Li M, Mechler BM, Delius H, Hoppe D, Stannek P, Walter C, Glinka A and Niehrs C: Kremen proteins are Dickkopf receptors that regulate Wnt/beta-catenin signalling. Nature 417(6889): 664-667, 2002. PMID: 12050670. DOI: 10.1038/nature756
30 Cselenyi CS and Lee E: Context-dependent activation or inhibition of Wnt-beta-catenin signaling by Kremen. Sci Signal 1(8): pe10, 2008. PMID: 18314504. DOI: 10.1126/stke.18pe10

31 Carmon KS, Gong X, Yi J, Wu L, Thomas A, Moore CM, Masuho I, Timson DJ, Martemyanov KA and Liu QJ: LGR5 receptor promotes cell-cell adhesion in stem cells and colon cancer cells via the IQGAP1-Rac1 pathway. J Biol Chem 292(36): 14989-15001, 2017. PMID: 28739799. DOI: 10.1074/jbc.M117.786798

32 Jang JW, Kim MK and Bae SC: Reciprocal regulation of YAP/TAZ by the Hippo pathway and the Small GTPase pathway. Small GTPases 11(4): 280-288, 2020. PMID: 29457552. DOI: $10.1080 / 21541248.2018 .1435986$

33 Park HW, Kim YC, Yu B, Moroishi T, Mo JS, Plouffe SW, Meng Z, Lin KC, Yu FX, Alexander CM, Wang CY and Guan KL: Alternative Wnt signaling activates YAP/TAZ. Cell 162(4): 780794, 2015. PMID: 26276632. DOI: 10.1016/j.cell.2015.07.013

34 Lian Z, Liu J, Li L, Li X, Clayton M, Wu MC, Wang HY, Arbuthnot P, Kew M, Fan D and Feitelson M: Enhanced cell survival of Hep3B cells by the hepatitis B $\mathrm{x}$ antigen effector, URG11, is associated with upregulation of beta-catenin. Hepatol 43(3): 415-424, 2006. PMID: 16496348. DOI: 10.1002/hep.21053

35 Du R, Xia L, Sun S, Lian Z, Zou X, Gao J, Xie H, Fan R, Song J, Li X, Liu J and Fan D: URG11 promotes gastric cancer growth and invasion by activation of beta-catenin signalling pathway. J Cell Mol Med 14(3): 621-635, 2010. PMID: 19413886. DOI: 10.1111/j.1582-4934.2008.00622.x

36 Kuchenbaecker KB, Ramus SJ, Tyrer J, Lee A, Shen HC, Beesley J, Lawrenson K, McGuffog L, Healey S, Lee JM, Spindler TJ, Lin YG, Pejovic T, Bean Y, Li Q, Coetzee S, Hazelett D, Miron A, Southey M and Chenevix-Trench G: Identification of six new susceptibility loci for invasive epithelial ovarian cancer. Nat Genet 47(2): 164-171, 2015. PMID: 25581431. DOI: $10.1038 / \mathrm{ng} .3185$

37 Phelan CM, Kuchenbaecker KB, Tyrer JP, Kar SP, Lawrenson K, Winham SJ, Dennis J, Pirie A, Riggan MJ, Chornokur G, Earp MA, Lyra PC Jr, Lee JM, Coetzee S, Beesley J, McGuffog L, Soucy P, Dicks E, Lee A and Pharoah PDP: Identification of 12 new susceptibility loci for different histotypes of epithelial ovarian cancer. Nat Genet 49(5): 680-691, 2017. PMID: 28346442. DOI: $10.1038 / \mathrm{ng} .3826$
Received August 21, 2020

Revised September 12, 2020 Accepted September 16, 2020 